\title{
Spinal Cord Injury
}

National Institute of Neurological Disorders and Stroke (NINDS)

\section{Source}

National Institute of Neurological Disorders and Stroke (NINDS). Spinal Cord Injury

Information Page.

A spinal cord injury usually beg ins with a sudden, traumatic blow to the spine that fractures or dislocates vertebrae. The damage begins at the moment of injury when displaced bone fragments, disc material, or ligaments bruise or tear into spinal cord tissue. Most injuries to the spinal cord don't completely sever it. Instead, an injury is more likely to cause fractures and compression of the vertebrae, which then crush and destroy axons -- extensions of nerve cells that carry signals up and down the spinal cord between the brain and the rest of the body. An injury to the spinal cord can damage a few, many, or almost all of these axons. Some injuries will allow almost complete recovery. Others will result in complete paralysis 